\title{
Laparoscopic Partial Nephrectomy for Renal Angiomyolipoma: A Retrospective Cohort Study
}

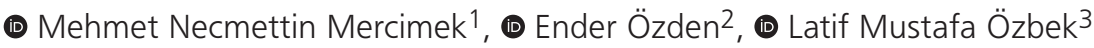 \\ 'Samsun Liv Hospital, Clinic of Urology, Samsun, Turkey \\ 2 Ondokuz Mayıs University Faculty of Medicine, Department of Urology, Samsun, Turkey \\ 3 Iğdır State Hospital, Clinic of Urology, Iğdır, Turkey
}

\begin{abstract}
Objective: The aim of this study is to investigate the surgical and functional outcomes of laparoscopic partial nephrectomy (LPN) in patients with renal angiomyolipoma (RAML).

Materials and Methods: From April 2014 to February 2018, the data of a total of 346 patients who underwent LPN were retrospectively reviewed. Twentyone patients with final pathology report indicating RAML were included in the study. Demographic data, operative indications, intraoperative and postoperative outcomes as well as complications were evaluated.

Results: Eighteen female (85.7\%) and 3 male (14.3\%) patients were included in this study. The mean age was $55.14 \pm 11.95$ years. Of the RAMLs, $52.4 \%$ were in the right kidney and $47.6 \%$ in the left kidney. The mean tumor size was $31.38 \pm 16.72 \mathrm{~mm}$ and the mean RENAL Nephrometry score was $5.90 \pm 1.51$ (4-10). RAML could not be differentiated from RCC in 12 patients (57.1\%) by preoperative computer tomography and/or magnetic resonance images. In 4 patients, LPN was implemented by off-clamp technique whereas, in 17 patients it was implemented by global ischemia technique. The mean operation duration was $67.86 \pm 20.77$ (40-110) minutes ( $\mathrm{min}$ ) and the mean estimated blood loss was 117.14 \pm 59.7 (30-220) milliliters. In 17 patients who underwent LPN by global ischemia technique, the mean warm ischemia time was 10.88 \pm 5.19 (6-22) min. According to the Modified Clavien-Dindo classification, grade 1 complications were detected in 3 patients, postoperatively. The most considerable renal functional changes were detected between preoperative and postoperative- $1^{\text {st }}$ day levels. However, at the end of the first year, the difference was found to decrease.

Conclusion: LPN is a safe and effective treatment option for the treatment of RAMLs that are not able to be distinguished from renal cell carcinoma radiologically. Besides the definitive treatment, the exact pathological diagnosis can be ensured by LPN.
\end{abstract}

Keywords: Angiomyolipoma, laparoscopy, partial nephrectomy

\section{Introduction}

Angiomyolipoma (AML) is the most prevalent benign mesenchymal tumor of the kidney consisting of varying proportion of adipose tissue, blood vessel, and smooth muscle cells. The majority of AMLs occur in sporadic form with 4 times more in women than men however up to $20 \%$ of those may be accompanied by lymphangioleiomyomatosis (LAM) and tuberous sclerosis complex (TSC), which is an exceptional autosomal-dominant disorder related to over activation of the mammalian target of rapamycin pathway (1). The hereditary forms of RAMLs tend to be multifocal and bilateral in contrast to sporadic form which is more often specified as a solitary lesion (2).
The wide use of radiological imaging has increased the incidental detection of renal masses. The main considerable role of imaging is to differentiate RAMLs from renal cell carcinoma (RCC). As, the fat content of AML decreases, it is able to be difficult to differentiate it from RCC by computerized tomography (CT) and magnetic resonance image (MRI). It has been stated that approximately $5 \%$ of AMLs have devoid of adipose tissue to be identified in imaging. Therefore, biopsy or surgical resection may be needed to acquire the correct diagnosis (3).

Clinically, the majority of small AMLs are asymptomatic. However, increase in tumor size over time may lead to symptoms including flank pain, hematuria, palpable mass, retroperitoneal

Cite this article as: Mercimek MN, Özden E, Özbek LM. Laparoscopic Partial Nephrectomy for Renal Angiomyolipoma: A Retrospective Cohort Study. Bull Urooncol 2020;19(2):74-79 
hemorrhage as well as shock. The indication for an active intervention of RAMLs has been recommended for patients with symptoms, tumor size $>4 \mathrm{~cm}$, suspicion of malignancy and for women of childbearing age. Treatment approaches include a wide range of options ranging from radical nephrectomy or partial nephrectomy to minimally invasive ablative therapies (4). In this retrospective study, we aimed to evaluate the indications, intraoperative and postoperative outcomes in patients with RAML who underwent laparoscopic partial nephrectomy (LPN) in a tertiary care institution.

\section{Materials and Methods}

This was a single center retrospective cohort study and was conducted in compliance with the ethical principles of the Helsinki Declaration. Ethics approval was granted by the Ondokuz Mayıs University Clinical Research Ethics Committee (OMU KAEK 2019/157, February 14 ${ }^{\text {th }}, 2019$ ). Informed consent was obtained from all patients prior to their database inclusion. The current study was designed as a retrospective chart review of patients who underwent LPN from April 2014 to February 2018. The data of 21 patients who underwent LPN with final pathology report of AML were included in the study. Surgical indications were the presence of tumors that were indistinguishable from RCC on radiological imaging, increased tumor size, and/or changes in imaging characteristics in RAMLs $\geq 4 \mathrm{~cm}$ on follow-up and preference of surgery instead of selective artery embolization (SAE) by the patients. The patients who were managed by radical nephrectomy, open partial nephrectomy, active surveillance, SAE, and ablative therapies including cryoablation and radiofrequency ablation (RFA) were also excluded in this study. All patients were evaluated before the active surgical intervention with ultrasonography (USG), and CT or MRI. All LPNs were performed by a single surgeon (E.O) in a tertiary care institution via transperitoneal or retroperitoneal access which was previously described in the literature $(5,6)$.

Follow-up consisted of measuring renal functions including serum creatinine $(\mathrm{Cr})$ and estimated glomerular filtration rate (eGFR) using the Chronic Kidney Disease Epidemiology Collaboration equation in the first-month postoperatively and 3 months thereafter (7). The initial radiologic evaluation by CT was performed in the $3^{\text {rd }}$ month after surgery. USG was used as an imaging modality only if there were normal anatomical findings existed. CT was performed when necessary (8).

Demographic variables and tumor characteristics including age, gender, symptoms, comorbities, serum $\mathrm{Cr}$, and eGFR, hemoglobin $\mathrm{Hb}$ level, American Society of Anesthesiologists score, tumor size, laterality, radiologic features, and RENAL Nephrometry score were recorded. Peroperative and postoperative data, including duration of operation, type of the ischemia, warm ischemia time (WIT), estimated blood loss, complications, postoperative $\mathrm{Cr}$, $\mathrm{Hb}$, eGFR, hospitalization, surgical margin status and final pathology report were also recorded. Demographic data, operative indications, peroperative and postoperative outcomes and complications were evaluated. Postoperative complications were also categorized according to the Modified Clavien-Dindo classification system. Moreover, trifecta was defined as negative surgical margin, WIT shorter than 20 min and complication free recovery.

\section{Statistical Analysis}

Patients' data were expressed as mean \pm standard deviation (minimum-maximum). The data were analyzed using Statistics Package for Social Sciences version 24 (IBM SPSS®), Armonk, NY). The functional outcomes including serum $\mathrm{Cr}$ and eGFR were evaluated by two-way ANOVA. Hb values were compared with Paired-samples $t$ test. A $p$ value $<0.05$ was considered significant.

\section{Results}

The study included 18 women (85.7\%) and 3 men (14.3\%) patients whose age range was 29 to 71 years. The mean age was $55.14 \pm 11.95$ years. While the renal masses were detected incidentally by routine radiological imaging in 20 patients (95.2\%), only one patient with tumor size $50 \mathrm{~mm}$ and RENAL nephrometry score 8 , (4.8\%) presented with hematuria. All patients had only one renal mass, $52.4 \%$ of the masses were in the right kidney and $47.6 \%$ in the left kidney. The mean tumor size was $31.38 \pm 16.72 \mathrm{~mm}$ and the mean RENAL Nephrometry score was $5.90 \pm 1.51(4,5,6,7,8,9,10)$. Hypertension was the most frequently detected comorbidity. Seven patients (33.3\%) were receiving antihypertensive therapy. Nonetheless, one patient (4.8\%) had Diabetes Mellitus and the other one (4.8\%) also had coronary artery disease. Demographic data of patients are shown in Table 1.

In the surgical indication analysis, symptomatic RAML was determined in one patient (4.8\%). While surgical intervention was performed in 8 patients (38.1\%) due to tumor size $\geq 4 \mathrm{~cm}$, inability to differentiate RAMLs from RCC in 12 patients (57.1\%) by preoperative CT and/or MR images. LPN indications for RAMLs are shown in Table 2. Our general clinical management of RAMLs that have been radiologically confirmed as requiring active intervention is primarily the application of SAE. However, we applied LPN in patients who were indistinguishable from RCC radiologically or whose tumor diameter enlarged and

\begin{tabular}{|l|l|}
\hline \multicolumn{2}{|l|}{ Table 1. Demographic data of patients } \\
\hline Age, years (min-max) & $55.14 \pm 11.95(29-71)$ \\
\hline $\begin{array}{l}\text { Gender, } \mathbf{n}(\%) \\
\text { Female }\end{array}$ & $18(85.7 \%)$ \\
Male & $3(14.3 \%)$ \\
\hline Comorbidity, $\mathbf{n}(\%)$ & \\
Hypertension & $7(33.3 \%)$ \\
Diabetes Mellitus & $1(4.8 \%)$ \\
Coronary Artery disease & $1(4.8 \%)$ \\
\hline Characteristics of the tumor & \\
>4 cm & 7 \\
$\leq 4$ cm & 14 \\
Mean size, mm & $31.38 \pm 16.72(10-60)$ \\
Polar & 15 \\
Central & 6 \\
RENAL score, mean (range) & $5.9 \pm 1.5(4-10)$ \\
\hline ASA score & $9(42.9 \%)$ \\
ASA 1 & $12(51.7 \%)$ \\
ASA 2 & \multicolumn{2}{|l}{} \\
\hline ASA: American society of anesthesiologists, Min: Minimum, Max: Maximum, n: \\
Number
\end{tabular}


radiological image characteristics changed. Another indication for LPN was the patient's preference.

While 18 (85.7\%) patients underwent transperitoneal approach, 3 (14.3\%) patients underwent retroperitoneoscopic partial nephrectomy (RPN). In 4 patients, LPN was implemented by offclamp technique whereas, in 17 patients it was implemented by global ischemia technique. The mean duration of operation was $67.86 \pm 20.77$ (40-110) minute ( $\mathrm{min})$, the mean estimated blood loss was $117.14 \pm 59.7$ (30-220) milliliters (mL). In 17 patients who underwent LPN with global ischemia technique, the mean WIT was $10.88 \pm 5.19$ (6-22) min. There was no intraoperative complication however; postoperative complication took place in 3 patients. Two patients were closely followed up in terms of Hemaglobin drop (grade 1), postoperatively. Yet, there was no need for blood transfusion in these patients. The drain of the third patient was withdrawn on the $4^{\text {th }}$ postoperative day due to the prolonged drainage (grade 1). There was also no need for additional treatment or medication in that patient and only follow-up was enough. The mean hospital stay was $2.76 \pm 0.62$ (2-5) days. All surgical specimens were revealed as AML in the final pathology result. However, the surgical margin was reported as positive in only 1 patient (4.8\%). Trifecta was achieved in $18(85.7 \%)$ patients. The mean follow-up was $43.0 \pm 26.0$ (12-88) months. Intraoperative and postoperative outcomes are shown in Table 3.

In functional analysis, the mean preoperative eGFR value and postoperative $1^{\text {st }}$ day, $1^{\text {st }}$ month, $6^{\text {th }}$ month and $1^{\text {st }}$ year eGFR values were $102.46 \pm 23.31,78.64 \pm 19.47,83.11 \pm 18.93$, $87.39 \pm 21.39$, and $93.30 \pm 21.65$, respectively. Furthermore, the mean preoperative serum $\mathrm{Cr}$ level and postoperative $1^{\text {st }}$ day, $1^{\text {st }}$ month, $6^{\text {th }}$ month and $1^{\text {st }}$ year serum $\mathrm{Cr}$ levels were $0.70 \pm 0.13$, $0.88 \pm 0.15,0.83 \pm 0.14,0.80 \pm 0.13$ and $0.75 \pm 0.12$, respectively.

Table 2. Indication for laparoscopic partial nephrectomy

\begin{tabular}{|l|l|l|l|}
\hline & $\mathbf{n}$ & $\%$ & \multirow{2}{*}{ p value $^{\mathbf{a}}$} \\
\hline Hematuria & 1 & 4.8 & \multirow{2}{*}{$0.012^{*}$} \\
\cline { 1 - 3 } Suspicion of RCC & 12 & 57.1 & \\
\cline { 1 - 3 } Size $\geq 4 \mathrm{~cm}$ & 8 & 38.1 & \\
\cline { 1 - 2 } a: Chi-square test, *statistically significant. RCC: Renal cell carcinoma & \\
\hline
\end{tabular}

Table 3. Intraoperative variables and postoperative complications

\begin{tabular}{|l|l|}
\hline Side (R/L), $\mathrm{n}(\%)$ & $10(47.6 \%) / 11(52.4 \%)$ \\
\hline $\begin{array}{l}\text { Transperitoneal } \\
\text { Retroperitoneal }\end{array}$ & $18(85.7 \%)$ \\
\hline Global ischemia & $3(14.3 \%)$ \\
Off-clamp (zero ischemia) & $17(81 \%)$ \\
\hline Operation time, min & $4(19 \%)$ \\
\hline Estimated blood Loss, $\mathrm{mL}$ & $67.86 \pm 20.77(40-110)$ \\
\hline Warm ischemia time, min, $(\mathrm{n}=17)$ & $117.14 \pm 59.7(30-220)$ \\
\hline $\begin{array}{l}\text { Postoperative complications* } \\
\text { Hb drop (grade1), } \mathrm{n}\end{array}$ & $10.88 \pm 5.19(6-22)$ \\
Prolonged drainage (grade 1), $\mathrm{n}$ & $2(9.5 \%)$ \\
Total & $1(4.8 \%)$ \\
\hline
\end{tabular}

Min: Minute, mL: milliliters, R: Eight, L: Left, ${ }^{*}$ Clavien-Dindo classification, $\mathrm{n}$ : Number
Both the changes in eGFR and serum $\mathrm{Cr}$ values were found to be statistically significant in all variance. $P$ values were $p=0.001$, and $p=0.001$, respectively. Figure 1 shows eGFR changes and Figure 2 shows serum $\mathrm{Cr}$ changes according to time.

\section{Discussion}

It has been stated in a population-based screening study with ultrasound in Japan that RAMLs are not common in the general population and overall rate has been reported as $0.13 \%$ in adults. It is also specified that $\% 80$ of RAMLs are detected sporadically, whereas $20 \%$ of RAMLs are related to TSC (4). The sporadic form of RAML tends to be solitary renal mass. In general, RAMLs have a female predominance. The vast majority of patients are asymptomatic. Nonetheless, the most frightening complication due to RAML is retroperitoneal hemorrhage that is called Wunderlich syndrome. This clinical condition may be sudden, life-threatening as well as painful. It is thought that micro and macro aneurysms that correlated to an increase in tumor size may cause retroperitoneal bleeding (9). Hematuria, flank pain, and palpable renal mass are the other symptoms related to RAMLs (10). Moreover, it has been reported as an exceptional condition that epithelioid AML may behave like a malignant tumor that causes vena cava thrombus (11). In this study, $18(85.7 \%)$ of the 21 patients were female. Only 1 of the 21 patients had symptoms. In this 24-year-old female patient, the main complaint was an unclear loin pain and the detection of microscopic hematuria on urinalysis was the primary finding.

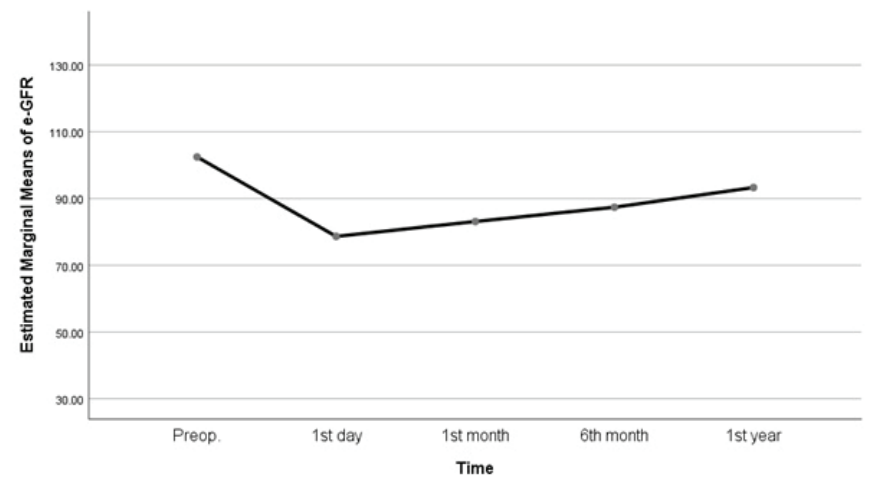

Figure 1. The changes in the mean eGFR values according to time eGFR: Estimated glomerular filtration rate

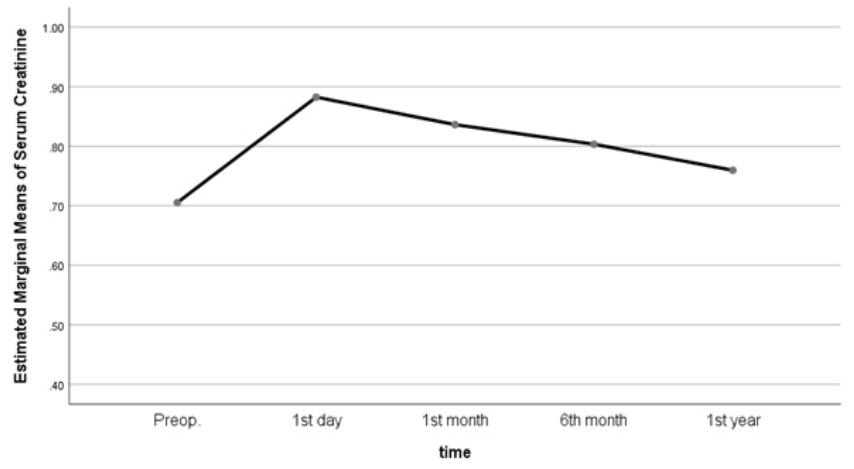

Figure 2. The changes in the mean serum creatinine values according to time 
Although the tumor size smaller than $4 \mathrm{~cm}$, LPN was applied upon the patient's preference.

Due to the widespread use of radiological imaging methods, renal masses are being detected earlier. It is declared that CT has a higher sensitivity and specificity to differentiate RAMLs from other renal malignant tumors. However, approximately $5 \%$ of RAMLs have devoid of adipose tissue. This is a challenging condition to distinguish oncocytoma and RAMLs with low-fat content from other malignant renal tumors by $\mathrm{CT}$ and MRI since fat-free RAMLs can easily imitate $\operatorname{RCC}(3,12,13)$. In the present study, RAMLs could not be differentiated from RCC in 12 patients $(57.1 \%)$ by radiology.

Asymptomatic patients and patients with tumor size $<4 \mathrm{~cm}$ RAMLs can be candidates for active surveillance. However, the indications for an active intervention for RAMLs are symptomatic masses, suspicion of malignancy, tumor size $>4 \mathrm{~cm}$, and women of childbearing age. Besides, patients with RAMLs with aneurysm $>5 \mathrm{~mm}$, patients with poor access to follow-up, patients with RAMLs associated with TSC, and patients with retroperitoneal hemorrhage are also candidates for an active intervention and patient's preference can also be an indication for an active intervention $(4,14)$. The most considerable reason for the suggestion of LPN as an active intervention in our cohort was inability to differentiate RAMLs from RCC on radiologic imaging. The second significant reason for applying LPN in the patients was that tumor size was $\geq 4 \mathrm{~cm}$ in 8 patients (38.1\%).

In recent years, advance in technology has increased the preference of nephron-sparing minimally invasive approaches including SAE (15), laparoscopic or robot assisted LPN $(16,17)$, as well as laparoscopic or percutaneous cryoablation or RFA in the treatment of RAMLs $(18,19)$. The aims of nephron-sparing approaches are relief of symptoms, preventing life-threatening acute hemorrhagic complications, preserving functional renal parenchyma, and also providing long-term kidney functions (20).

Given the effectivity of SAE, it is especially considered as a firstline treatment option for the management of RAMLs in patients with acute retroperitoneal bleeding, refractory hemodynamic instability or asymptomatic large RAMLs. However, it has been stated that the need for secondary intervention is high owing to recurrent hemorrhage, persistent symptoms or increased tumor diameter $(21,22)$. It is also stated that this condition may be related to large tumor size and etiology (23). The ratio of secondary intervention including re-embolization and need for surgery were declared in the literature as $9.1-64.7 \%$ and 2.1-35.3\%, respectively (24). Moreover, post-embolization syndrome characterized by fever, loin pain, vomiting or nausea, is the most common complication related to SAE and occurs in $85 \%$ of patients (25). In this study, patients who underwent SAE and were followed up in our clinic were not included in the study. In the vast majority of patients who underwent SAE RAML had been diagnosed radiologically before the intervention. However, in 12 patients (57.1\%) who underwent LPN RAML could not be differentiated from RCC by preoperative radiological imaging.

It has been stated that open or laparoscopic nephron-sparing surgery (NSS) for the management of renal tumors up to $4 \mathrm{~cm}$ in size in appropriate patients is a safe and effective treatment modality. Furthermore, it is stated that laparoscopic NSS is a safe and effective approach in the treatment of renal tumors larger than $4 \mathrm{~cm}$ (26). These recommendations may also be available for nephron-sparing treatment of RAMLs.

In the literature, one of the largest series of open NSS for the treatment of RAMLs was reported by Boorjian et al. (27) In this study, 58 patients with the mean tumor size of $3.9 \mathrm{~cm}(0.8$ 12) underwent open NSS with favorable functional results. It was stated that overall complication rate was $12.1 \%$, the mean estimated blood loss was $350 \mathrm{~mL}$ (50-1200), and the recurrence rate was $3.4 \%$. In a similar study, Minervini et al. (28) declared that the increase in tumor size was associated with higher intraoperative blood loss, longer ischemia time, and longer hospitalization. Despite the innovations in surgical technique, increase in surgical experience, and technological advances; intraoperative bleeding and WIT are still challenging parameters for LPN in the management of RAMLs. In recent years, it has been declared in a retrospective study that the implementation of SAE before LPN shortens duration of operation (93.7 vs 62.5 min), prolongs WIT (35.5 vs $21.6 \mathrm{~min}$ ) as well as increases intraoperative blood loss (170 vs $40 \mathrm{~mL}$ ) in patients with large $(>8 \mathrm{~cm}$ ) RAMLs (29). The outcomes of patients who underwent LPN with the diagnosis of either RCC or RAML were evaluated in two retrospective studies by Simmons et al. (30) $(n=35)$ and by Msezane et al. (31) $(n=14)$. It was stated that there was no difference in terms of duration of operation, blood loss, WIT and positive surgical margin. In a recent study reported by Liu et al. (8), data of 41 patients who underwent retroperitoneoscopic PN with the diagnosis of RAML were evaluated retrospectively. The median tumor size was $11.4 \mathrm{~cm}(8-23 \mathrm{~cm})$. In 35 patients, the surgery ended up laparoscopically. Conversions to open surgery and to nephrectomy were $9.8 \%$ and $4.9 \%$, respectively. The median duration of operation, WIT, and EBL were $167 \mathrm{~min}, 21 \mathrm{~min}$, and $200 \mathrm{~mL}$, respectively. Intraoperative complications occurred in $5 \%$ and postoperative complications occurred in $17.1 \%$ of patients. Furthermore, intraoperative blood transfusion was required in 8 patients.

In this study, unlike other studies cited above, a standard surgical approach was not applied to the patients. The transperitoneal or retroperitoneal approach was preferred according to the localization, size, RENAL score of the renal mass as well as the surgeon's preference. In addition, the non-ischemic LPN was performed in 4 patients according to the preoperative tumor and kidney anatomy and vasculature. The mean tumor size was $31.38 \pm 16.72 \mathrm{~cm}(10-60 \mathrm{~cm})$. The mean duration of operation , WIT and EBL were $67.86 \pm 20.77 \mathrm{~min}, 10.88 \pm 5.19 \mathrm{~min}$, and $117.14 \pm 59.7 \mathrm{~mL}$, respectively. There was no need to convert to open surgery in any patient.

In this study, functional results of nephron-sparing PN in patients with RAML were found to be similar with previous literature $(8,28,29)$. In a systematic review, preoperative renal function, the preserved renal parenchymal volume, WIT, tumor size, age, gender, and comorbidity have been specified as factors affecting functional outcomes after PN. Moreover, preservation of remnant renal parenchyma has also been remarked as more substantial than WIT (32). In this study, the decrease in eGFR and increase in serum $\mathrm{Cr}$ values were found 
to be more prominent on the first postoperative day compared to preoperative values. The mean difference in eGFR was 23.82 $\mathrm{mL} / \mathrm{min}$ [95\% confidence interval $(\mathrm{Cl})$ (11.04-36.01)] when preoperative eGFR value and first postoperative day eGFR valuewere compared. Moreover, at the end of the $1^{\text {st }}$ year, the mean difference was $9.1 \mathrm{~mL} / \mathrm{min}$ [95\% Cl (1.06-17.26)].

\section{Study Limitations}

The limitations of this study included small number of subjects, retrospective analysis of patients' data as well as consisting of only one group. Evaluation of preoperative and postoperative split renal functions on the affected kidney would have given precious information in terms of functional outcomes. However, the evaluation of renal functions with renal scintigraphy in patients who underwent PN was not being used in our routine practices. Therefore, a prospective study might be needed for this reason.

\section{Conclusion}

Minimally invasive PN is a safe and effective treatment option for the treatment of RAMLs that are not able to be distinguished from RCC radiologically, that are symptomatic and/or large in size $(>4 \mathrm{~cm})$.

\section{Acknowledgements}

Publication: The results of the study were not published in full or in part in form of abstracts.

Contribution: There is not any contributors who may not be listed as authors.

Conflict of Interest: No conflict of interest was declared by the authors.

Financial Disclosure: The authors declared that this study received no financial support.

\section{Ethics}

Ethics Committee Approval: Ethics approval was granted by the Ondokuz Mayıs University Clinical Research Ethics Committee (OMU KAEK 2019/157, February 14th, 2019).

Informed Consent: Informed consent was obtained from all patients.

Peer-review: Externally peer-reviewed.

\section{Authorship Contributions}

Concept: M.N.M., E.Ö., L.M.Ö., Design: M.N.M., E.Ö., Data Collection or Processing: M.N.M., E.Ö., L.M.Ö., Analysis or Interpretation: M.N.M., E.Ö., Literature Search: M.N.M., L.M.Ö., Writing: M.N.M., E.Ö., L.M.Ö.

\section{References}

1. Bhatt JR, Richard PO, Kim NS, et al. Natural History of Renal Angiomyolipoma (AML): Most Patients with Large AMLs $>4 \mathrm{~cm}$ Can Be Offered Active Surveillance as an Initial Management Strategy. European urology 2016;70:85-90.

2. Delhorme JB, Fontana A, Levy A, et al. Renal angiomyolipomas: At least two diseases. A series of patients treated at two European institutions. European journal of surgical oncology : the journal of the European Society of Surgical Oncology and the British Association of Surgical Oncology 2017;43:831-836.

3. Park BK. Renal Angiomyolipoma: Radiologic Classification and Imaging Features According to the Amount of Fat. AJR American journal of roentgenology 2017;209:826-835.

4. Flum AS, Hamoui N, Said MA, et al. Update on the Diagnosis and Management of Renal Angiomyolipoma. The Journal of urology 2016;195:834-846.

5. Singh D, Rubenstein M, Gill IS. Laparoscopic partial nephrectomy. Journal of endourology 2005; 19:451-455; discussion 455.

6. Gill IS, Rassweiler JJ. Retroperitoneoscopic renal surgery: our approach. Urology 1999;54:734-738.

7. Levey AS, Stevens LA. Estimating GFR using the CKD Epidemiology Collaboration (CKD-EPI) creatinine equation: more accurate GFR estimates, lower CKD prevalence estimates, and better risk predictions. American journal of kidney diseases : the official journal of the National Kidney Foundation 2010;55:622-627.

8. Liu X, Ma X, Liu Q, et al. Retroperitoneal laparoscopic nephron sparing surgery for large renal angiomyolipoma: Our technique and experience. A case series of 41 patients. International journal of surgery 2018;54:216-221.

9. Chronopoulos PN, Kaisidis GN, Vaiopoulos CK, et al. Spontaneous rupture of a giant renal angiomyolipoma-Wunderlich's syndrome: Report of a case. International journal of surgery case reports 2016; 9:140-143.

10. Lienert AR, Nicol D. Renal angiomyolipoma. BJU international 2012;110 Suppl 4:25-27.

11. Cavicchioli FM, D'Elia C, Cerruto MA, et al. Giant bilateral renal angiomyolipomas: a case report. Urologia internationalis 2014;92:366-368.

12. Ljungberg $B$, Albiges $L$, Abu-Ghanem $Y$, et al. European Association of Urology Guidelines on Renal Cell Carcinoma: The 2019 Update. European urology 2019;75:799-810.

13. Sureka B, Khera PS. Radiologic Classification and Imaging Features of Renal Angiomyolipomas According to the Amount of Fat. AJR American journal of roentgenology 2018;210:W136.

14. Mues AC, Palacios JM, Haramis G, et al. Contemporary experience in the management of angiomyolipoma. Journal of endourology 2010;24:1883-1886.

15. Muller A, Rouviere O. Renal artery embolization-indications, technical approaches and outcomes. Nature reviews Nephrology 2015;11:288-301.

16. Husillos Alonso A, Subira Rios D, Bolufer Moragues E, et al. Laparoscopic partial nephrectomy with prior superselective embolisation as treatment for giant renal angiomyolipoma. Actas urologicas espanolas 2018;42:64-68.

17. Kara O, Akca O, Zargar H, et al. Robotic Partial Nephrectomy in the Treatment of Renal Angiomyolipoma. Journal of endourology 2016;30:275-279.

18. Makki A, Graumann O, Hoyer S, et al. Cryoablation of Renal Angiomyolipoma: An Evaluation of Safety and Efficacy. Journal of endourology 2017;31:1117-1122.

19. Castle SM, Gorbatiy V, Ekwenna O, et al. Radiofrequency ablation (RFA) therapy for renal angiomyolipoma (AML): an alternative to angio-embolization and nephron-sparing surgery. BJU international 2012;109:384-387.

20. Sivalingam S, Nakada SY. Contemporary minimally invasive treatment options for renal angiomyolipomas. Current urology reports 2013;14:147-153.

21. Ramon J, Rimon U, Garniek A, et al. Renal angiomyolipoma: longterm results following selective arterial embolization. European urology 2009;55:1155-1161.

22. Villalta JD, Sorensen MD, Durack JC, et al. Selective arterial embolization of angiomyolipomas: a comparison of smaller and larger embolic agents. The Journal of urology 2011;186:921-927. 
23. Eijkemans MJ, van der Wal W, Reijnders LJ, et al. Long-term Follow-up Assessing Renal Angiomyolipoma Treatment Patterns, Morbidity, and Mortality: An Observational Study in Tuberous Sclerosis Complex Patients in the Netherlands. American journal of kidney diseases : the official journal of the National Kidney Foundation 2015;66:638-645.

24. Bardin F, Chevallier O, Bertaut A, et al. Selective arterial embolization of symptomatic and asymptomatic renal angiomyolipomas: a retrospective study of safety, outcomes and tumor size reduction. Quantitative imaging in medicine and surgery 2017;7:8-23.

25. Wang $C$, Yang $M$, Tong $X$, et al. Transarterial embolization for renal angiomyolipomas: A single centre experience in 79 patients. The Journal of international medical research 2017;45:706-713.

26. Caputo PA, Zargar H, Ramirez D, et al. Cryoablation versus Partial Nephrectomy for Clinical T1b Renal Tumors: A Matched Group Comparative Analysis. European urology 2017;71:111-117.

27. Boorjian SA, Frank I, Inman B, et al. The role of partial nephrectomy for the management of sporadic renal angiomyolipoma. Urology 2007;70:1064-1068.
28. Minervini A, Giubilei G, Masieri L, et al. Simple enucleation for the treatment of renal angiomyolipoma. BJU international 2007;99:887-891.

29. Qin C, Wang Y, Li P, et al. Super-Selective Artery Embolization before Laparoscopic Partial Nephrectomy in Treating Renal Angiomyolipoma. Urologia internationalis 2017;99:277-282.

30. Simmons MN, Chung BI, Gill IS. Perioperative efficacy of laparoscopic partial nephrectomy for tumors larger than $4 \mathrm{~cm}$. European urology 2009;55:199-207.

31. Msezane L, Chang A, Shikanov S, et al. Laparoscopic nephronsparing surgery in the management of angiomyolipoma: a single center experience. Journal of endourology 2010;24:583-587.

32. Klatte T, Ficarra V, Gratzke C, et al. A Literature Review of Renal Surgical Anatomy and Surgical Strategies for Partial Nephrectomy. European urology 2015;68:980-992. 